\title{
THE THURSTON NORM AND 2-HANDLE ADDITION
}

\author{
MARTIN SCHARLEMANN
}

\begin{abstract}
Suppose a 2-handle is attached to a compact orientable 3-manifold $M$ along an annulus $A$ contained in a subsurface $N$ of $\partial M$. If $N$ is compressible in $M$, but $N-A$ is not, then the Thurston norm is unaffected. This generalizes a series of results due to Przytycki, Jaco, and Johannson.
\end{abstract}

Following Thurston [Th], define the complexity $\chi_{-}(S)$ of an oriented surface $S$ to be $-\chi(C)$, where $C$ is the union of all nonsimply connected components of $S$ and $\chi(C)$ is its Euler characteristic. Hence $\chi_{-}(S) \geq 0$. For $M$ a compact oriented 3-manifold and $N$ a (possibly empty) surface in $\partial M$, assign to any homology class $\alpha$ in $H_{2}(M, N ; \mathbf{Z})$ the minimum complexity $\nu$ of all oriented imbedded surfaces whose fundamental class represents $\alpha$. Thurston shows that there is a unique continuous extension of $\nu$ to $\mathrm{H}_{2}(M, N ; \mathbf{R})$. It is a pseudo-norm and, if the fundamental class of every embedded sphere, torus, disk, and annulus is trivial in $H_{2}(M, N)$, it is a norm.

Any continuous map $\varphi:(X, Y) \rightarrow(M, N)$ induces a pseudo-norm $\varphi^{*}(\nu)$ on $H_{2}(X, Y)$ defined by $\varphi^{*}(\nu)(\alpha)=\nu\left(\varphi_{*}(\alpha)\right)$. If $\varphi:\left(M^{\prime}, N^{\prime}\right) \rightarrow(M, N)$ is an inclusion of 3-manifolds, then clearly $\varphi^{*}(\nu) \leq \nu^{\prime}$.

Let $M^{+}$be the 3-manifold obtained from $M$ by attaching a 2-handle to $M$ along an annulus $A$ in $N$. This alters $N$ by a surgery to yield a surface $N^{+}$in $\partial M^{+}$. Inclusion induces an epimorphism $\varphi: H_{2}(M, N-A) \rightarrow H_{2}\left(M^{+}, N^{+}\right)$.

THEOREM. Let $M^{+}$be the 3-manifold obtained from a compact oriented 3manifold $M$ by attaching a 2-handle along an annulus $A$ in a (possibly empty) surface $N$ in $\partial M$. If $N$ is compressible but $N-A$ is not, then $\varphi^{*}\left(\nu^{+}\right)=\nu$.

As a special case we have the main theorem of [Joh]:

COROLLARY. Suppose $M^{+}$, is obtained from the $\partial$-reducible 3-manifold $M$ by attaching a 2-handle along an annulus $A$ for which $\partial M-A$ is incompressible. If there is an incompressible, nonseparating surface $S^{+}$in $M^{+}$then there is an incompressible nonseparating surface $S$ in $M^{+}$which is entirely contained in $M$ and with complexity less than $S^{+}$.

Proof OF THE Corollary. Let $\alpha^{+}$be the fundamental class of $S^{+}$in $H_{2}\left(M^{+}, \partial M^{+}\right)$. Since $\varphi$ is an epimorphism, there is a class $\alpha$ in $H_{2}(M, \partial M-A)$ such that $\varphi(\alpha)=\alpha^{+}$. By the Theorem, $\nu(\alpha)=\nu^{+}\left(\alpha^{+}\right) \leq \chi_{-}\left(S^{+}\right)$, so there is a surface $S$ in $M$ which is homologous to $S^{+}$(and hence nonseparating in $M^{+}$) and with $\chi_{-}(S) \leq \chi_{-}\left(S^{+}\right)$.

Received by the editors May 22, 1985.

1980 Mathematics Subject Classification (1985 Revision). Primary 57N10; Secondary 57M35.

Research supported in part by a National Science Foundation Grant. 
REMARKS. Johannson defines complexity of a surface $S$ somewhat differently (and less naturally) as $\beta_{1}(S)+\operatorname{genus}(S)$. Although his theorem is not then an immediate consequence, it does follow from the proof.

Note that $M$ and $M^{+}$may be reducible. This complicates the proof somewhat. The clearest (but long) proof would have three stages: First show that $N^{+}$is incompressible. Second, show that any sphere in $M^{+}$is cobordant to a sphere in $M$. This would allow us (by reducing $M$, which has no effect on the norm) to ignore spheres in $M^{+}$as well. Finally, cobord any surface in $M^{+}$to one in $M$ of no higher complexity.

The first stage was carried out by Przytycki $[\mathbf{P}]$ and Jaco $[\mathbf{J}]$. This is redone in [Sc] using an "outermost fork" argument which shows that a compression disk for $N^{+}$in $M^{+}$can be "squeezed" into $M$. This squeezing operation may not be an isotopy, but it is always a cobordism and so preserves homology class. Moreover, the proof does not require that the surface being squeezed is a disk. The Theorem will be proven by applying the same argument at the other two stages. For the sake of brevity, both stages have been compressed into one. This slightly complicates the argument (and accounts for the special treatment accorded surfaces which are unions of spheres).

PROOF OF THEOREM. Let $\left(S^{+}, \partial S^{+}\right)$be an oriented surface properly imbedded in $\left(M^{+}, N^{+}\right)$. Isotope $\partial S^{+}$in $N^{+}$so it is disjoint from the 2-handle $E$. By general position we can assume that $S^{+} \cap E$ consists of $n$ disks parallel to the core of $E$. Define the augmented complexity of $S^{+}$as follows: If $S^{+}$is a disjoint collection of spheres, let the augmented complexity be the ordered pair $(-1, n)$. If $S^{+}$has some component which is not a sphere, define the augmented complexity to be the ordered pair $\left(\chi_{-}\left(S^{+}\right), n\right)$.

DEFINITION. The augmented norm of $\mu$ in $\mathrm{H}_{2}\left(\mathrm{M}^{+}, \mathrm{N}^{+} ; \mathbf{Z}\right)$ is the minimal augmented complexity (via lexicographical order) of all surfaces in $\left(M^{+}, N^{+}\right)$whose fundamental class represents $\mu$.

LEMMA. The augmented norm always has second component 0.

In other words, if $\mu$ in $\mathrm{H}_{2}\left(\mathrm{M}^{+}, \mathrm{N}^{+} ; \mathbf{Z}\right)$ is the fundamental class of a disjoint collection of spheres in $\mathrm{M}^{+}$, then it is also the fundamental class of a disjoint collection of spheres in $M$. In the general case there is always a surface $S$ in $M$ whose fundamental class is $\mu$ for which $\chi_{-}(S)=\nu^{+}(\mu)$.

PROOF OF THEOREM FROM LEMMA. It suffices to show $\nu(\alpha) \leq \nu^{+}(\varphi(\alpha))$ for each $\alpha$ in $H_{2}(M, N-A)$. Applying the Lemma to $\varphi(\alpha)$, there is an oriented surface $(S, \partial S)$ lying in $(M, N-A) \subset\left(M^{+}, N^{+}\right)$whose fundamental class $\alpha^{+}$in $H_{2}\left(M^{+}, N^{+} ; \mathbf{Z}\right)$ satisfies $\alpha^{+}=\varphi(\alpha)$ and with $\chi_{-}(S)=\nu^{+}(\varphi(\alpha))$. Since $S$ lies in $M$, we may also regard $\alpha^{+}$as a class in $H_{2}(M, N-A)$ and observe that $\alpha-\alpha^{+}$ is in $\operatorname{ker}(\varphi)$. But $\operatorname{ker}(\varphi)$ is the cyclic group generated by the fundamental class of the annulus $(A, \partial A)$ so $\nu(\operatorname{ker}(\varphi))=0$. Hence

$$
\nu(\alpha) \leq \nu\left(\alpha-\alpha^{+}\right)+\nu\left(\alpha^{+}\right)=\nu\left(\alpha^{+}\right) \leq \chi_{-}(S)=\nu^{+}(\varphi(\alpha)) .
$$

ProOF OF LEMMA. Pick a homology class $\mu$ in $H_{2}\left(M^{+}, N^{+} ; \mathbf{Z}\right)$ and let $\left(S^{+}, \partial S^{+}\right)$be an oriented surface, properly imbedded in $\left(M^{+}, N^{+}\right)$, whose fundamental class is $\mu$ and whose augmented complexity is the augmented norm on $\mu$. Suppose $S^{+}$intersects $E$ in $n$ disks. Inductively assume that the Lemma is true for every homology class of lower augmented norm. 
ASSERTION 1. If $\left(S^{+}, \partial S^{+}\right)$is the disjoint union of two surfaces, each with augmented complexity less than $S^{+}$, then $n=0$.

ProOF. Suppose $S$ is the disjoint union of $S_{1}$ and $S_{2}$. If each has augmented complexity less than that of $S^{+}$then, by inductive assumption, their fundamental classes $\mu_{1}$ and $\mu_{2}$ are represented by (not necessarily disjoint) surfaces in $(M, N-A)$ with no greater augmented complexity than those of $S_{1}$ and $S_{2}$. It is easy to check that if $\mu_{1}$ and $\mu_{2}$ are each represented in $M$ by disjoint spheres, then so is $\mu_{1}+\mu_{2}$. In the general case,

$$
\nu\left(\mu_{1}+\mu_{2}\right) \leq \nu\left(\mu_{1}\right)+\nu\left(\mu_{2}\right) \leq \chi_{-}\left(S_{1}\right)+\chi_{-}\left(S_{2}\right)=\chi_{-}\left(S^{+}\right)=\nu^{+}(\mu)
$$

so $\mu=\mu_{1}+\mu_{2}$ is represented in $(M, N-A)$ by a surface of complexity $\nu^{+}(\mu)$.

We can assume there is no compressing disk ( $N$-compressing disk) for $S^{+}$contained entirely in $M$ (resp. $(M, N))$, for such a compression would increase neither the complexity nor $n$ (and would decrease the former unless $\chi\left(S^{+}\right)=0$ ). Denote by $p_{0}$ and $p_{n+1}$ the two components of $\partial A$ and by $E_{0}$ and $E_{n+1}$ the disks they bound in $N^{+}$. Denote by $\left\{p_{1}, \ldots, p_{n}\right\}$ the simple closed curves of $S^{+} \cap A$ in order, so that, for $0 \leq i \leq n, p_{i}$ and $p_{i+1}$ together bound a subannulus $A_{i}$ of $A$. For $1 \leq i \leq n$, denote by $E_{i}$ the disk in $S^{+}$which $p_{i}$ bounds. Denote by $S$ the surface $S^{+} \cap M$.

Case 1. $S$ is compressible in $M$.

ProOF. Let $F$ be a compressing disk for $S$ in $M$. Since $F$ is not a compressing disk for $S^{+}, \partial F$ divides $S^{+}$into two components (one of which is a disk, but neither is a disk disjoint from $E$ ). Then a regular neighborhood of $S^{+} \cup F$ is a cobordism between $S^{+}$and two disjoint surfaces $S_{1}$ and $S_{2}$, each of augmented complexity less than that of $S^{+}$, whose union has augmented complexity equal to that of $S^{+}$. The proof now follows from Assertion 1 applied to $S_{1} \cup S_{2}$.

Case 2. The general case.

Consider an arbitrary $N$-compressing disk $F$ for $S$ in $M$. Then $\operatorname{int}(F)$ and $F$ are disjoint, $\partial F=\alpha \cup \beta$ where $\alpha$ is an arc which is essential in $S, \beta$ is an arc in $N$, and $\alpha \cap \beta$ consists of two points in $\partial S$. Isotope $\beta$ rel $\partial$ so that it intersects $\partial A$ minimally.

ASSERTION 2. Either

(a) $\beta$ is not contained in $A$ and has one end on $p_{1}$, and the other on $p_{n}$.

(b) There is a disk in $N^{+}$whose boundary is the union of $\beta \cap N^{+}$and an arc of $\partial A$.

(c) $n=0$.

PROOF. If $(\beta, \partial \beta)$ is inessential in $(N, \partial S)$, then the union along $\beta$ of $F$ and the disk in $N$ bounded by $\beta$ and an $\operatorname{arc}$ of $\partial S$ is a compressing disk for $S$, and (c) follows from Case 1 .

If both ends of $\beta$ lie on the same component $c$ of $\partial S^{+}$, then a regular neighborhood of $S^{+} \cup F$ is a cobordism of $S^{+}$to the surface $S^{\prime}$ obtained by compressing $S^{+}$ along $F$. But since $S^{+}$is of minimal augmented complexity in its homology class, $\alpha$ and some subarc $\gamma$ of $c$ bound a disk in $S^{+}$. Since $\alpha$ is essential in $S$, any such disk must pass through the 2-handle $E$. Hence $S^{\prime}$ is the union of two pieces, $S_{1}$ and $S_{2}$, each with augmented complexity less than that of $S^{+}$. The proof follows from Assertion 1.

If both ends of $\beta$ lie on the same component $p_{i}$ of $\partial S-\partial S^{+}$and $(\beta, \partial \beta)$ is essential in $(N, \partial S)$ then $\beta$ does not lie in $A$, and $i=1$ or $n$. Say $i=1$. Then 
$\beta \cap A$ consists of two arcs running from one end to the other of the annulus $A_{0}$. Denote by $B_{1}$ and $B_{2}$ the two bands into which the ends of $\beta$ divide $A_{0}$. Then the union of $F$ and $B_{1}$ along $\beta \cap A_{0}$ is an annulus $Q$ in $M$ with one end in $N^{+}$and one end in $S^{+}$. If the end of $Q$ in $N^{+}$is inessential, then the disk it bounds in $N^{+}$ satisfies (b). If the end of $Q$ in $N^{+}$is essential, then so is the end of $Q$ in $S^{+}$, since $N^{+}$is known to be incompressible. In particular, $S^{+}$is not a union of spheres. A regular neighborhood of $S^{+} \cup Q$ is then a cobordism from $S^{+}$to a surface $S^{\prime}$ of no greater augmented complexity. Now the component of $E-S^{+}$containing $A_{0}$ may be viewed as an isotopy $\operatorname{rel} \beta \cap A_{0}$ from $B_{1}$ to $B_{2}$. Perform this isotopy on the piece of $S^{\prime}$ lying on one side of $B_{1}$. The effect is to remove the disk in $E$ bounded by $p_{1}$ from $S^{\prime}$. In particular, we have reduced the augmented complexity of $S^{\prime}$, which is impossible.

If the ends of $\beta$ lay on different components of $\partial S^{+}$, then $F$ would be a boundarycompressing disk for $S^{+}$contained in $M$, which has already been ruled out.

If one end of $\beta$ lies in $\partial S^{+}$and one in $\partial S$ then the disk $F$ provides a track for an isotopy of $S^{+}$which reduces $n$ by one, which is impossible. If the $\operatorname{arc} \beta$ of $F \cap \partial D$ lies in the annulus $A_{i}$ bounded by $p_{i}$ and $p_{i+1}$, the disk $F$ provides a track for an isotopy of $S^{+}$which reduces $n$ by two-again impossible. The only remaining alternative is precisely (a) above.

Suppose $c$ is a simple closed curve in $N$ which intersects $\left\{p_{i}\right\}$ minimally. Then $c \cap A$ has the same number of boundary points on $p_{0}$ as on $p_{n}$ so $c \cap N^{+}$is a collection of arcs in $N-A$ which also has the same number of boundary points on $p_{0}$ as on $p_{n}$.

ASSERTION 3. There is no disk in $N^{+}$with boundary the union of an arc $\gamma$ in $\partial A$ and an $\operatorname{arc} \beta$ of $c \cap N^{+}$.

ProOF. An innermost such disk must contain one of $E_{0}$ or $E_{n+1}$ (say $E_{0}$ ) in its interior, since $c$ has minimal intersection with $\left\{p_{i}\right\}$. But then any arc of $c \cap N^{+}$ with one end at $p_{0}$ lies inside the disk and so must have other end at $p_{n}$. Since there are arcs with both ends in $p_{n}$ (e.g. $\beta$ ), this would imply there are more end points of $c \cap N^{+}$in $p_{n}$ than in $p_{0}$.

Let $D$ be a compression disk for $N$ in $M$, transverse to $S$, chosen to minimize the number of components of $D \cap S$. This assures in particular that each arc of $\partial D \cap A$ runs from one end of the annulus $A$ to the other, intersecting $\left\{p_{0}, \ldots, p_{n+1}\right\}$ in order.

ASSERTION 4. If any component of $D \cap S$ is a simple closed curve, then $n=0$.

PROOF. Such a simple closed curve cannot bound a disk in $S$, for an innermost such disk in $S$ could be substituted for a disk in $D$, reducing the number of intersections of $D$ with $S$. Hence, if there is a simple closed curve then $S$ is compressible. The assertion follows from Case 1.

ASSERTION 5. No component of $D \cap S$ is an inessential arc on $S$.

PROOF. If there is an inessential arc in $S$, there is a subdisk $Q$ of $S$ with interior disjoint from $D$ and boundary composed of two arcs, one in $D \cap S$ and the other in $\partial S$. Then the boundary of a regular neighborhood of $D \cup Q$ has three components. One of the two not parallel to $D$ is a compressing disk for $N$ with fewer components of intersection with $S$, a contradiction.

ASSERTION 6. Any outermost arc of $S \cap D$ in $D$ has one end in $p_{1}$ and one end in $p_{n}$. Moreover, the arc of $\partial D$ which it cuts off does not lie entirely in $A$. 
ProOF. By Assertion 5, the disk cut off by the outermost arc is an $N$-compressing disk for $S$. The assertion then follows from Assertions 2 and 3.

DEFinition. A disk $F$ in $M$ is an $i$-cycle if the interior of $F$ is disjoint from $S$ and, for some fixed $1 \leq i \leq n-1$, there is an orientation of $\partial F$ such that $\partial F$ consists alternately of $\operatorname{arcs}$ in $S$ and $A_{i}$ running from $p_{i}$ to $p_{i+1}$.

ASSERTION 7. There is no $i$-cycle in $M$.

ProOF. Lef $F$ be an $i$-cycle in $M$ such that $\partial F \cap A_{i}$ has $q$ arcs. Let $C$ be the component of $S^{+}$containing $p_{i}$ and $p_{i+1}$. (They lie on the same component since $q$ arcs of $\partial F$ lying in $S$ have one end in each.) The component of $E-S^{+}$containing $A_{i}$ may be viewed as a 1-handle $H$ attached to $C$ at $E_{i} \cup E_{i+1}$, providing a cobordism from $C$ to a connected surface $C^{\prime}$ with $\chi\left(C^{\prime}\right)=\chi(C)-2$. On the other hand, a relative neighborhood of $F$ may be viewed as a 2-handle attached to $C^{\prime}$ providing a cobordism from $C^{\prime}$ to a surface $C^{\prime \prime}$ with $\chi\left(C^{\prime \prime}\right)=\chi\left(C^{\prime}\right)+2=\chi(C)$. Moreover, $C^{\prime \prime}$ is still connected, since there is a circle in $C^{\prime}$ (e.g. $p_{i}$ ) which intersects $\partial F$ algebraically $q \geq 1$ times. Substitute $C^{\prime \prime}$ for $C$ in $S^{+}$to obtain a cobordant surface $S^{\prime \prime}$ with the same complexity (and still a collection of spheres if $S^{+}$was), but with two fewer components of intersection with $E$. Then the augmented complexity of $S^{\prime \prime}$ is less than that of $S^{+}$, a contradiction.

COMPLETION OF PROOF. The proof is completed by citing the argument of [Sc, §2]. A study of the intersection $\operatorname{arcs}$ of $D$ with $S$, using Assertion 6 guarantees that if $D \cap S$ is nonempty, there must be an $i$-cycle among the disk components of $D-S$. We conclude that $D \cap S$ is empty. But since $D \cap A$ is nonempty, this means $n=0$.

\section{REFERENCES}

[J] W. Jaco, Adding a 2-handle to a 3-manifold: an application to property $R$, Proc. Amer. Math. Soc. 92 (1984), 288-292.

[Joh] K. Johannson, On surfaces in one-relator 3-manifolds, preprint.

[P] J. Przytycki, Incompressible surfaces in 3-manifolds, Thesis, Columbia Univ., 1981.

[Sc] M. Scharlemann, Outermost forks and a theorem of Jaco, Contemp. Math., vol. 44, Amer. Math. Soc., Providence, R.I., 1985, pp. 189-193.

[Th] W. Thurston, A norm for the homology of 3-manifolds, preprint.

DePARTMENT OF MATHEMATICS, UNIVERsity OF CAlifornia, SANTA BaRbara, CALIFORNIA 93106 